\title{
Análisis de los Términos Utilizados en la Literatura Científi- ca para Referirse a los Ligamentos Extracapsulares de la Articulación Témporomandibular. Parte I: Ligamentum sphenomandibulare
}

\author{
A Study of Terms Used in Scientific Literature to Refer to the Extracapsular \\ Ligaments of the Temporomandibular Joint. Part I: Ligamentum sphenomandibulare
}

Ramón Fuentes ${ }^{*, * *}$; Cristina Bucchi ${ }^{*, * *}$ \& Mario Cantin ${ }^{*, * * * * * * * *}$

FUENTES, R.; BUCCHI, C. \& CANTIN, M. Análisis de los términos utilizados en la literatura científica para referirse a los ligamentos Extracapsulares de la articulación témporomandibular. Parte I: Ligamentum sphenomandibulare. Int. J. Morphol., 32(3):981-986, 2014.

RESUMEN: La Terminología Anatómica es la base de correcta comunicación anatómica y establece la unificación internacionalmente aceptada para diversas estructuras anatómicas. Sin embargo, su uso en la literatura pedagógica y científica no se ha aplicado por completo. El objetivo de esta investigación fue analizar la literatura en relación al uso correcto de los términos referentes al Ligamento esfenomandibular de la Articulación témporomandibular (ATM). Se realizó una revisión en libros de anatomía y artículos científicos relacionados y se comparó el uso de términos referentes al ligamento esfenomandibular con la Terminología Anatómica Internacional (TAI). Se encontraron diferencias en cuanto a los términos usados (sinonimia) para referirse tanto al ligamento esfenomandibular, así como a las estructuras que sirven de origen e inserción a este con los términos establecidos por la TAI en latín, inglés y algunos términos traducidos al español. La sinonimia y uso de epónimos encontrados dificultan la comunicación entre los científicos del área, así como el aprendizaje de los alumnos de pregrado y postgrado, cuya principal fuente de estudio continúan siendo los libros de anatomía.

PALABRAS CLAVE: Ligamento esfenomandibular; Ligamentum sphenomandibulare; Ligamento anterior del martillo; Ligamento discomaleolar; Terminología Anatómica Internacional.

\section{INTRODUCCIÓN}

La Terminología Anatómica es la base de la comunicación anatómica y establece la unificación internacionalmente aceptada para una serie de estructuras anatómicas. A fines del siglo XIX existían más de cincuenta mil términos anatómicos para referirse a cinco o seis mil estructuras del cuerpo humano (López \& Terrada, 1990). Con el propósito de generar univocidad de los términos anatómicos y facilitar la enseñanza y comunicación entre la comunidad científica, nace en 1895 la Nómina Anatómica en Basilea, que luego de siglos de modificaciones y ratificaciones, deriva a la actual Terminología Anatómica Internacional (TAI) (1998) y está en constante revisión, ya que en las ciencias de la salud se requiere de una terminología clara y precisa.
Aunque la terminología anatómica actual es el resultado de siglos de trabajo científico, en la práctica no se ha aplicado por completo, lo que resulta evidente con la lectura de algunos libros y artículos científicos que aún utilizan epónimos o varios nombres para una misma estructura. Así como en diversas estructuras, esto se observa en ligamentos extracapsulares de la articulación témporomandibular (ATM) como el Ligamentum sphenomandibulare.

El objetivo de esta investigación fue analizar la literatura científica de libros y artículos de anatomía humana, en relación al uso correcto de los términos referentes a descripción, clasificación, origen, inserción y función del ligamento esfenomandibular (Ligamentum sphenomandibulare) de la ATM.

\footnotetext{
*Programa Magister en Odontología, Facultad de Odontología, Universidad de La Frontera, Temuco, Chile.

** Centro de investigación CIMOFIR, Facultad de Odontología, Universidad de La Frontera, Temuco, Chile.

${ }^{* * * *}$ Programa de Doctorado en Ciencias Morfológicas, Centro de investigación CIMA. Facultad de Odontología, Universidad de La Frontera, Temuco, Chile.

${ }^{* * * * *}$ Centro de Investigación en Ciencias Biomédicas, Universidad Autónoma de Chile, Temuco, Chile.
} 


\section{MATERIAL Y MÉTODO}

Se realizó una revisión sistemática de la literatura en libros de anatomía y artículos científicos. Se incluyeron y analizaron 16 libros, pertenecientes a una colección particular, escritos en idiomas español, portugués e inglés y que trataban sobre la ATM. Se excluyeron los libros cuyo año de publicación fuese anterior a 1998 (año de publicación de la TAI). Se estudió específicamente lo referente a ligamento esfenomandibular (Ligamentum sphenomandibulare) y la conexión de este ligamento con el oído medio. Además, se realizó una búsqueda bibliográfíca de artículos en las bases PubMed, ScienceDirect y SciELO. Se utilizaron las palabras clave en español: Ligamento esfenomandibular, Ligamento esfenomaxilar, "Ligamento malar", Ligamento maleolar anterior, Ligamento anterior del martillo, Ligamento discomaleolar y Terminología Anatómica internacional; y en idioma inglés: Sphenomandibular ligament, Sphenomaxilar ligament, Anterior ligament of malleus e International Terminology. Se incluyeron artículos a texto completo, estudios observacionales realizados en humanos (adultos y fetos) y revisiones bibliográficas en español, inglés y portugués. Fueron excluidos artículos de opinión y cartas al editor.

En los artículos encontrados, se realizó la lectura de su resumen (en inglés o español), a fin de verificar su pertinencia. Tanto en los libros como los artículos seleccionados, se realizó la lectura y búsqueda manual de los términos referentes a descripción, clasificación, origen, inserción y función del ligamento esfenomandibular de la ATM. Los términos encontados fueron consignados en una ficha de recolección de datos; se compararon entre sí y con la TAI, en su versión latín-inglés, y la traducción literal de los términos del latín al español. No se utilizó la TAI con su versión traducida al español, debido a incompatibilidades y errores en la traducción al vernáculo español.

\section{RESULTADOS}

Denominación. El término más utilizado en la literatura fue ligamento esfenomandibular (español) (Giambartolomei, 2003; Bumann \& Lotzmann, 2000; Okeson, 2003; Wurgaft \& Montenegro, 2003; Maciel, 2003; Pertes \& Gross, 2005; Rubiano, 2005; Sosa, 2006; Katchburian \& Arana, 2004; Velayos \& Santana, 2001; Alves \& Candido, 2009; Lopes, 2004; Latarjet \& Ruiz, 2005; Isberg, 2003; Schunke et al., 2010). Diversos autores utilizaron más de un nombre para este ligamento (en conjunto con la denominación anterior): ligamento interespinoso (Lopes), ligamento timpanomandibular (Burch, 1966) y ligamento esfenomaxilar (Latarjet \& Ruiz; Giambartolomei). En los libros escritos en idioma español, sólo Velayos \& Santana utilizan el término en latín (Ligamentum sphenomandibulare), en conjunto con el término en español.

Clasificación. Diversos autores mencionan al ligamento esfenomandibular (Ligamentum sphenomandibulare) como uno de los dos ligamentos accesorios de la ATM (ligamentos esfenomandibular y estilomandibular) (Okeson; Wurgaft \& Montenegro; Maciel; Pertes \& Gross; Velayos \& Santana). Otros se refieren a él como uno de los tres ligamentos accesorios de la ATM (ligamentos esfenomandibular, estilomandibular y pterigomandibular) (Giambartolomei; Sosa; Lopes), ligamentos extrínsecos (Lopes; Latarjet \& Ruiz) o auxiliares de la ATM (Rubiano). Además, Giambartolomei lo menciona como ligamento accesorio, secundario o de acción indirecta.

Origen. Se encontraron diferencias en los términos, detalle de descripción y también de las estructuras que dan origen a este ligamento. El origen varía de menor a mayor detalle en el hueso esfenoides (Maciel), ala mayor del hueso esfenoides (Isberg), espina del hueso esfenoides (Okeson; Wurgaft \& Montenegro; Pertes \& Gross; Rubiano; Katchburian \& Arana; Alves \& Candido; Latarjet \& Ruiz) y lado externo de la espina del hueso esfenoides (sic) (Lopes).

Otros autores describen el origen del ligamento en los huesos temporal y esfenoides (en ambos). Giambartolomei describe su origen en la cara lateral de la "língula esfenoidal" (espina del esfenoides) y en la porción más medial de la fisura escamosotimpánica (Cisura de Glasser) (sic). Velayos \& Santana lo describen en la espina del hueso esfenoides y en la zona inmediatamente adyacente del hueso temporal. Bumman \& Lotzmann en la apófisis espinosa del hueso esfenoides en un tercio de los pacientes y en la mayoría se inserta también en la cápsula articular medial, en la fisura petrotimpánica o en el ligamento malar anterior (sic).

Conexión de la ATM con el oído medio. Se describe una conexión entre la ATM y el oído medio, dada por una estructura ligamentosa. Esta estructura, se describe por algunos autores como una continuación (Velayos \& Santana) o porción timpánica del ligamento esfenomandibular (Maciel) (Fig. 1). Otros mencionan que esta conexión se genera por la continuidad del ligamento esfenomandibular con el denominado ligamento anterior del martillo (LAM) (Isberg) (Fig. 2) o ligamento timpanomandibular (Laterjet \& Ruiz); o que el ligamento esfenomandibular se continuaría con la unión de dos ligamentos, el LAM y el ligamento discomaleolar (LDM) (Loughner et al., 1989). 


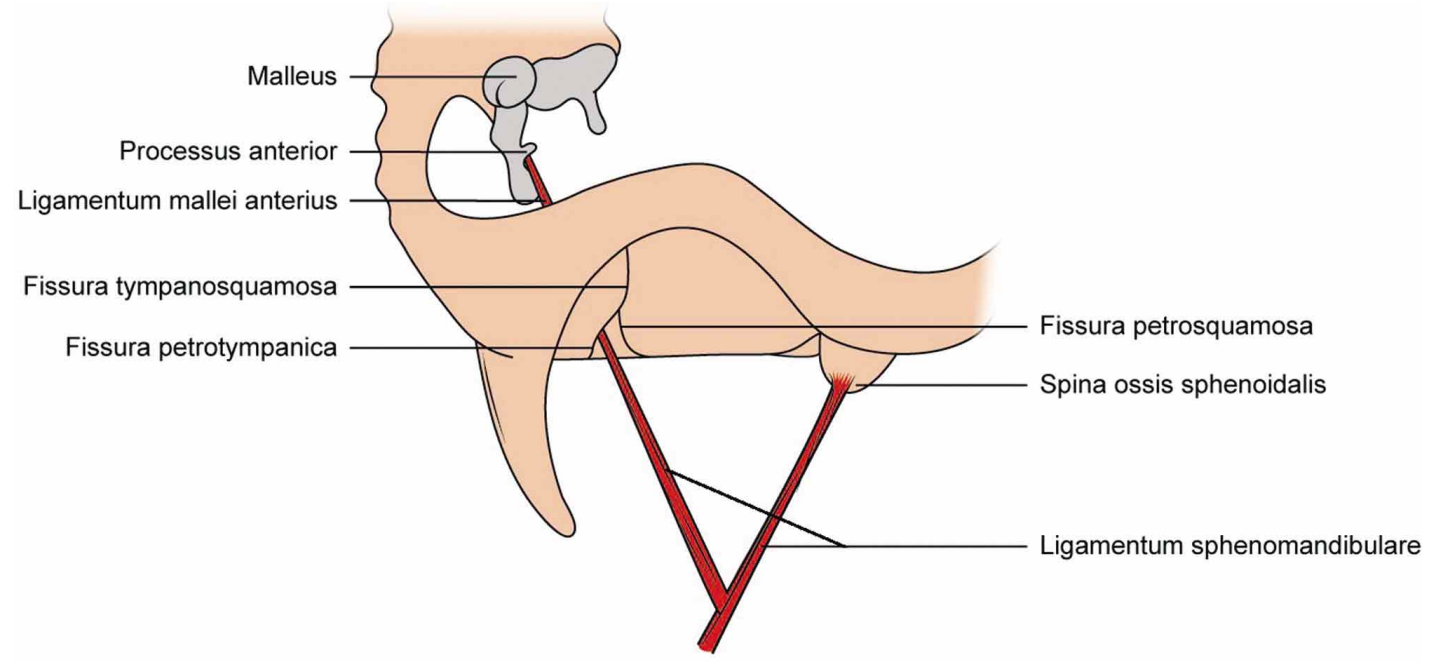

Fig. 1. Esquema de un corte parasagital del lado derecho del cráneo. Se puede observar que parte de las fibras del Ligamento esfenomandibular (Ligamentum sphenomandibulare) pasan a través de la Fisura petrotimpánica (Fissura petrotympania) y se insertan en el proceso anterior (Processus anterior) del martillo.

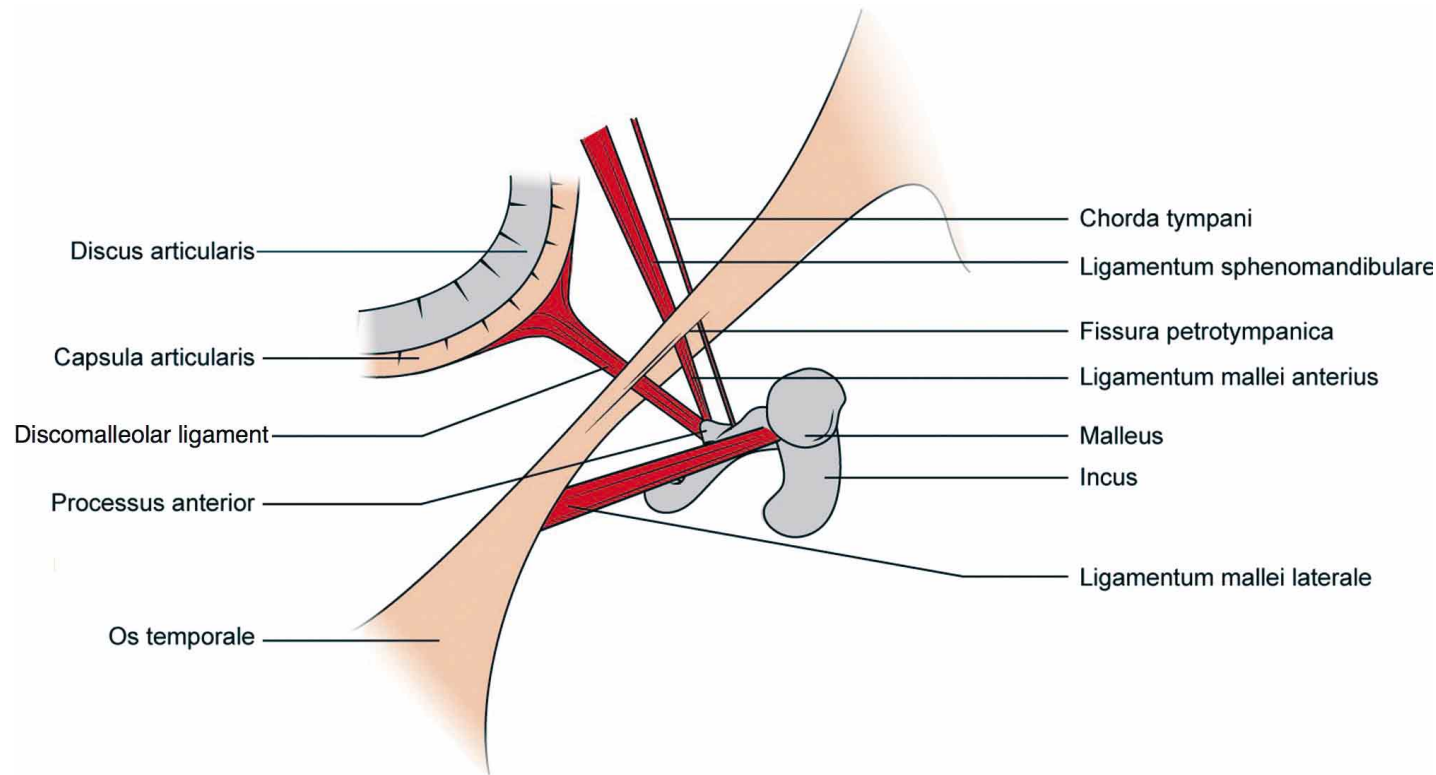

Fig. 2. Esquema de un corte transversal del oido medio y articulación témporomandibular del lado izquierdo del cráneo. Se observa la disposición del Ligamento discomaleolar (discomalleolar ligament), que conecta la capsula articular (Capsula articularis) con el martillo (Malleus) y del Ligamento esfenomandibular (Ligamentum sphenomandibulare) que cambia de nombre a Ligamento anterior del martillo (Ligamentum mallei anterius), al pasar por la Fisura petrotimpánica (Fissura petrotympania).

Inserción. Al igual que el origen, presenta una gran variabilidad de términos, descripción y estructuras relacionadas. La mayoría de los autores la describen en la língula mandibular (Okeson; Bumann \& Lotzmann; Wurgaft \& Montenegro; Rubiano; Alves \& Candido) o en la língula del foramen mandibular (sic) (Katchburian \& Arana); otros utilizan el término espina de Spix en conjunto con el de língula mandibular (Lopes; Latarjet \& Ruiz).
Otros autores describen con mayor detalle su inserción en la língula mandibular y el margen inferior del foramen mandibular (Pertes \& Gross). Velayos \& Santana lo describen en la espina de Spix (Lingula mandibulae), por anterior y posterior a ella. Maciel refiere su inserción en la rama mandibular, en su superficie medial, en la lingula de la rama ascendente mandibular (sic) (Isberg) y en la cara medial de la rama de la mandíbula, cerca del orificio superior del 
conducto dental (sic), en la língula de la mandíbula (Giambartolomei).

Función. Para Bumann \& Lotzmann y Wurgaft \& Montenegro el ligamento esfenomandibular cumple la función de impedir o limitar los movimientos de protrusión articular. Según Bumann \& Lotzmann impide los movimientos de mediotrusión, así como la apertura bucal pasiva. Para Sosa son complementarios para mantener la estabilidad mandibular, aunque recalca que su importancia es relativa para varios autores. Otros autores dan una importancia anatómica al ligamento el cual reforzaría la ATM (Maciel) o la parte posterior de la apófisis interpterigoides (sic) (Giambartolomei). Para Okeson y Pertes \& Gross no tiene efectos limitantes importantes o significativos en el movimiento mandibular.

\section{DISCUSIÓN}

Se encontraron diferencias en la literatura en cuanto a los términos usados para referirse al ligamento esfenomandibular y a las estructuras que sirven de origen e inserción a este, con los términos establecidos por la TAI. Según la TAI la denominación correcta en latín e inglés para este ligamento es Ligamentum sphenomandibulare y Sphenomandibular ligament, respectivamente; en español correspondería a ligamento esfenomandibular. Varios de los autores, usan términos obsoletos o más de un término para este ligamento, e incluso hay discordancia con respecto a que denotan los términos. Para Burch el ligamento timpanomandibular es sinónimo del ligamento esfenomandibular, mientras que para Latarjet \& Ruiz el ligamento timpanomandibular es la continuación del ligamento esfenomandibular hacia el oído medio.

La TAI clasifica los ligamentos de las articulaciones sinoviales del cuerpo humano en extracapsulares, capsulares e intracapsulares (siendo los extracapsulares los encontrados alejados de la capsula, los capsulares son un engrosamiento de la capsula y los intracapsulares se encuentran al interior de esta) (Paraskevas, 2011). El ligamento esfenomandibular se clasifica según Terminología dentro de los Ligamenta extracapsularia o extracapsular ligaments (términos en latín e inglés, respectivamente) y su denominación en español correspondería a ligamentos extracapsulares. Ninguno de los autores analizados lo clasifica de esta manera, ya que todos utilizan una clasificación funcional y no anatómica de los ligamentos de la ATM.

Por otra parte, la TAI define que los términos adecuados para referirse a las estructuras que sirven de origen al ligamento esfenomandibular son Os temporale, Os sphenoidales, Spina ossis sphenoidalis, Fissura tympanosquamosa, Fissura petrotympanica, Ala major y Capsula articularis, términos que en español se denominan hueso temporal, hueso esfenoides, espina del hueso esfenoides, fisura timpanoescamosa, fisura petrotimpanica, ala mayor (del esfenoides) y cápsula articular; la mayoría de los autores estudiados mencionan las estructuras de acuerdo a la TAI (Maciel; Isberg; Okeson; Wurgaft \& Montenegro; Pertes \& Gross; Rubiano; Katchburian \& Arana; Alves \& Candido; Latarjet \& Ruiz; Velayos \& Santana). Tradicionalmente en los textos se ha definido el origen o inserción superior del ligamento esfenomandibular en la espina del hueso esfenoides (Maciel, Okeson; Wurgaft \& Montenegro; Pertes \& Gross; Rubiano; Katchburian \& Arana; Alves \& Candido; Latarjet \& Ruiz, Lopes), aunque no existe acuerdo si es en la cara medial o lateral de esta. Otros lo definen en la espina del hueso esfenoides y en distintos puntos del hueso temporal (Giambartolomei, Velayos \& Santana) y muy pocos detallan la inserción superior en el oído medio (Velayos \& Santana; Maciel; Isberg; Laterjet \& Ruiz; Loughner et al.; Alves \& Deana, 2010).

En el año 1962 Pinto describe una estructura que hasta ese entonces, no estaba detallada en los libros de texto, denominándola "tiny ligament" (ligamento minúsculo) y la describe como una conexión que va desde cuello y proceso anterior del martillo hasta la parte medio-supero-posterior de la capsula articular, el disco articular y el ligamento esfenomandibular (ligmento de Pinto). Sin embargo, investigaciones realizadas sobre ATM de cráneos humanos muestran la existencia de dos estructuras ligamentosas distintas y conectadas que relacionarían la ATM con el oído medio, el LMA y LDM, que forman una estructura triangular donde el LDM constituye el margen lateral y el LMA el medial (Rodríguez-Vásquez et al., 1998; Ramírez et al., 2009). El LDM se inserta en el cuello del martillo o en algunos casos, en la cara lateral del LAM (Rowicki \& Zakrzewska, 2006) y pasa delante a la pared ótica anterior del oído medio y a través del extremo superior lateral de la fisura petrotimpánica, insertándose en la superficie medial del tejido retrodiscal y capsula de la ATM (Loughner). El LAM, se origina también en el proceso anterior del martillo (Ramírez et al.), pasa inferiormente a través de la fisura petrotimpánica y algunas de sus fibras se continuarían con las del ligamento esfenomandibular (Loughner). Esta conexión y sus relaciones anatómicas se encuentras esquematizadas en la Figura 2.

En un estudio realizado por Burch en el año 1966 sobre 63 ATM humanas, mostró que sólo seis tenían una inserción prominente en la espina del esfenoides y las 57 restantes mostraban su inserción en el área de la fisura 
petrotimpánica, además algunas fibras iban en sentido medial hacia la espina y en sentido lateral hacia la fisura tímpanoescamosa. En 13 de 63 muestras las fibras continuaban a través de la fisura hacia el martillo. Rodríguez-Vásquez et al. (1992) analizaron 60 fetos humanos y encontraron que el ligamento esfenomandibular se extendió e insertó en el proceso anterior del martillo. Según este autor el ligamento esfenomandibular tiene dos porciones claramente definidas, una yuxta-articular o extra-timpánica y otra timpánica, cuyo límite está definido por la fisura tímpano-escamosa. La inserción en la espina del esfenoides no fue observada en ningún caso. Esta diferencia anatómica entre LDM y el LMA estaría dada por su origen embriológico, el LDM corresponde al remanente primitivo del músculo pterigoideo lateral, mientras que el LMA es el remanente fibroso del cartílago mandibular (Cheynet et al., 2003), compartiendo su origen estructural con el ligamento esfenomandibular (Rodríguez-Vázquez et al., 1992). Con el desarrollo la fisura petrotimpánica se cierra, sirviendo de límite entre ambos ligamentos (Cheynet).

La TAI no incluye al ligamento discomaleolar, por ende, no es posible analizar el correcto uso del término, estableciendo su terminología desde su descripción en idioma inglés (discomalleolar ligament, Fig. 2). En cuanto al huesecillo del oído medio, el término correcto es Malleus (martillo) y su correspondiente Processus anterior (proceso anterior) y Ligamentum mallei anterius (ligamento anterior del martillo).
Según la TAI los términos correctos para referirse a las estructuras que sirven de inserción a este ligamento son; Lingula mandibulae, Foramen mandibulae y Ramus mandibulae, y en español corresponden a língula mandibular, foramen mandibular y rama mandibular. Aunque siete autores mencionan las estructuras de acuerdo a la TAI (Bumann \& Lotzmann; Okeson; Wurgaft \& Montenegro; Pertes \& Gross; Rubiano; Katchburian \& Arana; Alves \& Candido), no hay acuerdo si la inserción se produce en el vértice, margen inferior, posterior, o anterior de la língula mandibular.

La mayoría de la literatura científica revisada muestra diferencias en cuanto a los términos usados para referirse al Ligamentum sphenomandibulare y a las estructuras que sirven de origen e inserción a este, con los términos establecidos por la TAI. Existen también discrepancias con respecto a la función, clasificación y conexión de esta estructura con el oído medio, presentándose en este último caso, estructuras no descritas en la TAI. Esto dificulta la comunicación entre los estudiantes, profesionales y científicos del área, por lo que es necesario utilizar el criterio unificado y términos unívocos que la TAI ofrece, junto trabajar y sugerir la inclusión de las estructuras no presentes en ella a través de simposios locales para Terminología Anatómica y reuniones SILAT (Simposios Iberolatinoamericanos de Terminología) con representación ante la FIPAT (Federative International Programme for Anatomical Terminology).

FUENTES, R.; BUCCHI, C. \& CANTIN, M. A study of terms used in scientific literature to refer to the extracapsular ligaments of the temporomandibular joint. Part I: Ligamentum sphenomandibulare. Int. J. Morphol., 32(3):981-986, 2014.

SUMMARY: Terminología Anatómica is currently the international standard for anatomical terminology, and establishes an internationally accepted and unified nomenclature for several anatomical structures. However, International Anatomical Terminology (IAT) is not always used correctly in scientific literature. The purpose of this review is to examine scientific literature in order to determine whether the terms that refer to the sphenomandibular ligament of the temporomandibular joint (TMJ) are being used correctly. Scientific books and articles were reviewed, and the terms used were compared with those established by the IAT. Our study found differences between the terms actually used (Synonyms) to refer to the sphenomandibular ligament and to the anatomical structures where the ligament originates and where it is inserted and those established by the IAT in Latin, English and some terms translated into Spanish. The use of eponyms and synonyms found, creates difficulties in communication between scientists and in the learning process of undergraduate students, who frequently use textbooks.

KEY WORDS: Sphenomandibular ligament; Sphenomaxillary ligament; Anterior ligament of malleus; Discomalleolar ligament; Anatomical Terminology.

\section{REFERENCIAS BIBLIOGRÁFICAS}

Alves, N. \& Candido, P. L. Anatomía Aplicada a la Odontología. São Paulo, Santos, 2009.

Alves, N. \& Deana, N.F. Frequency of occurence of the discomalleolar ligament in the adult man. Int. J. Morphol., 28(2):515-518, 2010.
Bumann, A. \& Lotzmann, U. Atlas de Diagnóstico Funcional y Principios Terapeúticos en Odontología. Barcelona, Masson, 2000.

Burch, J. The cranial attachment of the sphenomandibular (tympanomandibular) ligament. Anat. Rec., 156(4):433-7, 1966. 
Cheynet, F.; Guyot, L.; Richard, O.; Layoun, W. \& Gola, R. Discomallear and malleomandibular ligaments: anatomical study and clinical applications. Surg. Radiol. Anat., 25(2) :152-7, 2003.

Giambartolomei, L. A. Anatomía del complejo articular cráneomandibular. Villa María, Red de editoriales de universidades nacionales, 2003.

Isberg, A. Disfunción de la Articulación Temporomandibular. Una Guía Práctica para el Profesional. $2^{\mathrm{a}}$ ed. São Paulo, Artes Médicas Latinoamerica, 2003.

Katchburian, E. \& Arana, V. Histología e Embriología Oral. $2^{\mathrm{a}}$ ed. São Paulo, Médica Panamericana, 2004.

Latarjet, M. \& Ruiz, L. Anatomía Humana. Tomo 2. $2^{\mathrm{a}}$ ed. Buenos Aires, Médica Panamericana, 2005.

Lopes, A. Anatomía Cabeça e Pescoço. Rio de Janeiro, GuanabaraKoogan, 2004.

Loughner, B.; Larkin, L. \& Mahan, P. Discomalleolar and Anterior Malleolar Ligaments: Possible Causes of Middle Ear Damage During Temporomandibular Joint Surgery. Oral Surg. Oral Med. Oral Pathol., 68(1):14-22, 1989.

Maciel, R.N. ATM e Dores Craneofaçiais. Fisiopatologia Básica. São Paulo, Santos, 2003.

Okeson, J. Tratamiento de Oclusión y Afecciones Temporomandibulares. 5a ed. Madrid, Mosby Inc., 2003.

Pertes, R. \& Gross, S. Tratamento Clínico das disfunçoes Temporomandibulares e da dor Orofacial. São Paulo, Quintessence, 2005

Paraskevas, G.K. Human ligaments classification: a new proposal. Folia Morphol. (Warsz), 70(2):61-7, 2001.

Pinto, O. A New Structure Related to the Temporomandibular Joint and Middle Ear. J. Prosthet. Dent., 12(1):95-103, 1962.

Ramírez, L.; Ballesteros, L. \& Sandoval, G. A Direct Anatomical study of the morphology and functionality of disco-malleolar and anterior malleolar ligaments. Int. J. Morphol., 27(2):36779, 2009.

Rodriguez-Vazquez, J.F.; Mérida-Velasco, J.R. \& Jiménez-Collado, J. Development of the human sphenomandibular ligament. Anat. Rec., 233(3):453-60, 1992.

Rodríguez-Vázquez, J.F.; Mérida-Velasco, J.R.; Mérida-Velasco, J.A. \& Jiménez-Collado, J. Anatomical considerations on the discomalleolar ligament. J. Anat., 192(Pt 4):617-21, 1998.

Rowicki, T. \& Zakrzewska, J. A study of the discomalleolar ligament in the adult human. Folia Morphol. (Warsz), 65(2):121-5, 2006.
Rubiano, C. M. Tratamiento con Placas y Corrección Oclusal por Tallado Selectivo. Bogotá, Amolca, 2005.

Schunke, M.; Schulte, E. \& Schumacher, U. Prometheus. Texto y Atlas de Anatomía, Tomo 3. $2^{\text {a }}$ ed. Buenos Aires, Médica Panamericana, 2010.

Sosa, G. E. Detección Precoz de los Desordenes Témporomandibulares. Caracas, Amolca, 2006.

Federative International Committee on Anatomical Terminologies (FICAT). Terminologia Anatomica. International Anatomical Terminology. New York, Thieme, 1998.

Velayos, J. L. \& Santana H. D. Anatomía de la Cabeza con Enfoque Odontoestomatológico. $3^{\mathrm{a}}$ ed. Madrid, Médica Panamericana, 2001.

Wurgaft, R. \& Montenegro, M. A. Desarrollo y Estructura de la Articulación Temporomandibular. Santiago, Servimpres, 2003.

\section{Dirección para Corresponcia: \\ Dr. Ramón Fuentes Fernández \\ Facultad de Odontología \\ Universidad de La Frontera \\ Manuel Montt 112 \\ Casilla 54-D, Temuco \\ CHILE}

Email: ramon.fuentes@ufrontera.cl

Recibido: 29-01-2014

Aceptado: 08-05-2014 